BERC-PHY-99/102

\title{
Limits to Sympathetic Evaporative Cooling of a Two-Component Fermi Gas
}

\author{
by \\ M. Crescimanno, C. G. Kaoy and R. Peterson \\ Physics Department \\ Berea College \\ Berea, Ky. 40404
}

August 1999

\begin{abstract}
We find a limit cycle in a quasi-equilibrium model of evaporative cooling of a two-component trapped fermion gas. The existence of such a limit cycle represents an obstruction to reaching the quantum ground state evaporatively. We show that evaporatively $\beta \mu \sim \mathcal{O}(1)$. We speculate that one may be able to cool an atomic fermi gas further by photoassociating dimers near the bottom of the fermi sea.
\end{abstract}


I. Introduction: The spectacular successes of laser cooling techniques in creating Bose-Einstein condensation $(\mathrm{BEC})$ in trapped dilute alkali vapors ${ }^{[1,2,3]}$ has stimulated efforts to form dilute nearly degenerate atomic fermion gases. Such systems undoubtedly have unique phenomenology, and since one can control the composition, densities and even scattering lengths in principle, they furnish a window to familiar phenomena (such as superconductivity, etc.) in unusual parameter regimes. ${ }^{[4,5,6,7,8]}$ A critical step in achieving BEC in dilute alkali vapors is evaporative cooling. In this note we describe limitations to the use of evaporative cooling for a harmonically trapped two-component fermi system.

Overall antisymmetry of the final state wavefunction forces the s-wave scattering amplitude for two-body collisions in a single component polarized fermi gas to vanish identically. However, in a system composed of two or more fermi species there can still be appreciable s-wave scattering amplitudes at low energies. Recent experimental observation of quantum statistical effects reducing the scattering frequency at low temperature has been reported in reference [9]. Sympathetic evaporative cooling in two-component Bose systems has been experimentally verified ${ }^{10}$. Aspects of the dynamics of sympathetic cooling in a two component fermi system have been discussed theoretically ${ }^{11,12}$, and recently achieved experimentally ${ }^{13}$.

We will show in a robust model that sympathetic cooling of trapped fermions is intrinsically limited to $\frac{\mu}{T} \sim \mathcal{O}(1)$ where $T$ is the temperature and $\mu$ is the chemical potential. Largely independent of trap and atomic parameters, this limit indicates that sympathetic evaporative cooling alone cannot achieve occupation probabilities in the trap single particle ground state characteristic of typical degenerate fermi systems (e.g. atomic nuclei and typical metals).

In summary we model evaporation as a succession of quasi-equilibrium states. We find that evaporation moves the chemical potential towards saturation at a fixed (non-zero) fraction of the evaporation energy. Lowering the evaporation energy in an attempt to cool further simply causes the fermi surface and temperature to both recede, thus not substantially increasing the occupation probability of the lowest single particle state.

We demonstrate this limiting behavior in a model with two regimes, one for which the evaporative energy scales of both fermion types are identical and the opposite extreme where one selectively only allows evaporation of one species from the trap. Rather than end on a pessimistic note, we conclude by speculating on a possible method for surmounting the difficulty of cooling an atomic fermi gas.

II. The Model: We aim to explain general features of the evaporative cooling of a two-component fermion gas without recourse to the details of dynamics and transport. In particular, trap lifetime and other timescales will play almost no role in our considerations.

As described above, we focus on the case of a two-fermion system cooling sympathetically by evaporation through interspecial two-body collisions only. As described in the introduction, this is a reasonable assumption as long as the density is low and the temperature is low enough that the amplitude for intraspecial scattering is small.

In the limit of interest for the first regime, we model the evaporation process as a succession of quasi-equilibrium states in which the distribution functions of both species are cut off at $E_{\text {evap }}$. Aspects of the thermodynamics of dilute harmonically trapped fermions without an energy cutoff are described in Ref.[5]. Furthermore, we ignore the effect of any other environmental fields 
(for example, trap fields), and assume them to be constant over the lifetime of the system. The average effect of the interactions between the different species is absorbed into a mean field term which we sweep into $\mu$ (see, for example, Ref.[18]). We further assume that particle number is not communicated between the species, and so their individual $\mu$ can differ. Interspecial scattering processes do communicate energy between species, and so we assume that both species are always a at common temperature

Consider evaporating both species at the same energy cut-off $E_{\text {evap }}$. We complete the analysis for this case and then turn to the opposite extreme where only one species evaporates. If both fermion species $a$ and $b$ are (nearly) the same mass then crossing symmetry equates the total rate for scattering into final state $|a, b\rangle$ with that of $|b, a\rangle$. This means that for the same evaporative cutoff, the rate at which particles of one species evaporates equals that of the other.

Finally, for simplicity, we model the evaporative process as one which always reduces the particle number by one and removes energy $E_{\text {evap }}$. In the model we develop we will ignore the contribution to the cooling that results from the interspecial mean field ${ }^{18}$. In the cases of interest in current experiments this interspecial mean field energy is expected to be very small compared with the other relevant energy scales (for example the fermi energy). Although this model of evaporation is a gross simplification, it becomes a progressively better approximation as the temperature drops, and we are confident it captures the main features of the evaporative process.

We approximate each component's scaled number and energy by cut-off equilibrium distribution functions

$$
N=\int_{0}^{1} \frac{x^{d} \mathrm{~d} x}{e^{\beta(x-\mu)}+1} \quad E=\int_{0}^{1} \frac{x^{d+1} \mathrm{~d} x}{e^{\beta(x-\mu)}+1}
$$

where $\beta$ and $\mu$ are respectively the inverse temperature and the chemical potential both made dimensionless by factors of $E_{\text {evap }}$. Further, $d$ depends on the actual spectrum of the trap, and for a three dimensional isotropic harmonic trap is 2 . We will keep the discussion rather general with respect to $d$, but use $d=2$ in all the graphs and particular conclusions below. While the actual value of $d$ does not substantially impact the nature of the conclusions that we draw, we do find that reducing $d$ (by, for example, significantly changing the aspect ratio of the trap) makes sympathetic evaporative cooling generally less effective. Finally in Eq.(1) $E$ is dimensionless (i.e. in units of $\left.E_{\text {evap }}\right)$ and we have suppressed some overall factors that depend on $E_{\text {evap }}$ and trap frequency.

We model evaporative cooling by simply following Eq.(1) through flow along

$$
\left(\begin{array}{l}
\mathrm{d} N \\
\mathrm{~d} E
\end{array}\right)=\left(\begin{array}{l}
-1 \\
-1
\end{array}\right) \mathrm{d} N
$$

By using symmetries of the scattering for fermion species of nearly equal mass for the case where both fermion species are being evaporated at the same energy $E_{\text {evap }}$, we find that the net effect on the individual distribution functions is encapsulated in Eq.(1) and Eq.(2) for each species separately (and so in what follows for this regime we suppress indices).

The resulting differential equations for $\beta$ and $\mu$ along this evaporative trajectory read,

$$
\begin{gathered}
\frac{\partial \beta}{\partial N}=\frac{1}{\operatorname{det}(M)}\left(-\left.\frac{\partial E}{\partial \mu}\right|_{\beta}+\left.\frac{\partial N}{\partial \mu}\right|_{\beta}\right) \\
\frac{\partial \mu}{\partial N}=\frac{1}{\operatorname{det}(M)}\left(\left.\frac{\partial E}{\partial \beta}\right|_{\mu}-\left.\frac{\partial N}{\partial \beta}\right|_{\mu}\right)
\end{gathered}
$$

where the determinant $\operatorname{det}(M)$ is given via

$$
\operatorname{det}(M)=\left.\left.\frac{\partial N}{\partial \beta}\right|_{\mu} \frac{\partial E}{\partial \mu}\right|_{\beta}-\left.\left.\frac{\partial N}{\partial \mu}\right|_{\beta} \frac{\partial E}{\partial \beta}\right|_{\mu}
$$


which, from the quasi-equilibrium distribution functions Eq.(1) we find $\operatorname{det}(M)>0$ for all $\beta, d$ and $\mu$. This positivity may be understood on general grounds via the connection between $\operatorname{det}(M)$ and the specific heat, $c_{V}$ at constant $N$,

$$
c_{V}=\frac{\beta^{2} \operatorname{det}(M)}{\left.\frac{\partial N}{\partial \mu}\right|_{\beta}}
$$

and by the fact that $\left.\frac{\partial N}{\partial \mu}\right|_{\beta}>0$ as a consequence of, for example, Eq.(1). The $\operatorname{det}(M)$ vanishes in the low temperature limit as $\sim \frac{\pi^{2} \mu^{4}}{3 \beta^{3}}$. See Figure 1 for an example of this behavior (for $d=2$ and $\mu=2 / 3)$.

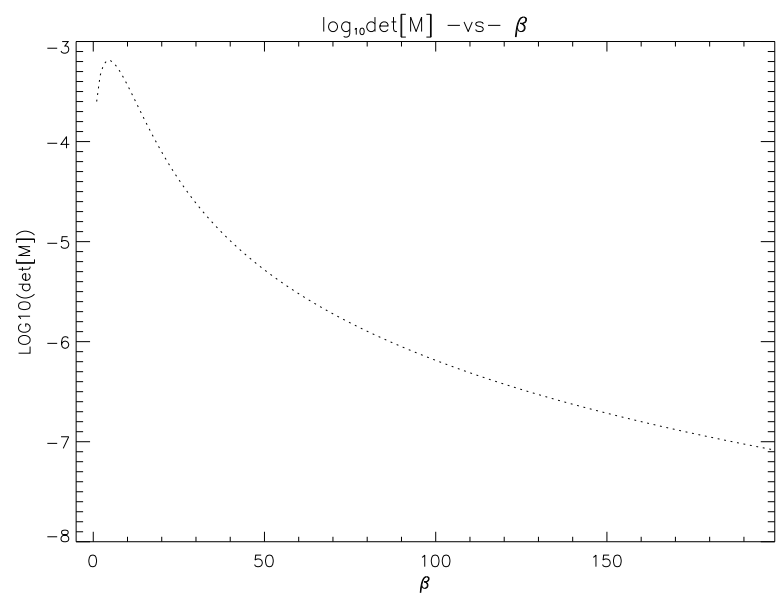

Figure 1: $\operatorname{det}(M)$ for $\mu=2 / 3$, as a function of $\beta$.

Starting far from degeneracy, the net effect of the evaporative process is to alter $\mu$ while increasing $\beta$. Note that using the equilibrium distributions Eq.(1) implies by Eq.(3) that $\beta$ increases monotonically along the flow. Upon reflection we see that, $\mu$ 's evolution does not share this property. Instead, we find that the system Eqs.(1), (3) and (4) has a limit cycle at $\left.\frac{\partial E}{\partial \beta}\right|_{\mu}-\left.\frac{\partial N}{\partial \beta}\right|_{\mu}=0$ in the $(\beta, \mu)$ plane. This limit cycle is at an intermediate value of $\mu=\mu^{*}(\beta)$ for all temperatures.

Figure 2 below is a graph of $\mu^{*}$ as a function of temperature for $d=2$

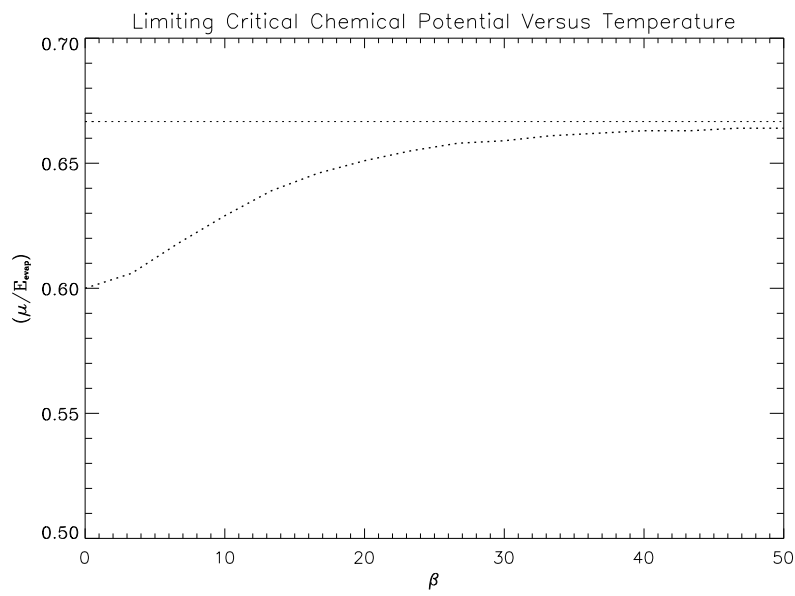

Figure 2: The Evaporative Limit Cycle with the Low-Temperature Asymptote

For $d=2$, the low temperature limit of $\mu^{*}$ is $2 / 3$. We reiterate, that for values of $(\beta, \mu)$ below the curve, the evaporative process increases $\mu$ whereas for $(\beta, \mu)$ values above the curve they reduce it. Thus, as one tries to lower the temperature lower by reducing $E_{\text {evap }}$ (thus increasing $\mu$ ), 
evaporation causes the fermi surface $\mu^{*} E_{\text {evap }}$ to also drop away. Of course, as one drops $E_{\text {evap }}$ the scaled inverse temperature $\beta$ also drops trivially.

A figure of merit measuring how close one is to the quantum many body ground state is the occupation probability in the trap single particle ground state. This is a function of the ratio of the chemical potential to the temperature, in our notation $\beta \mu$. This combination is independent of $E_{\text {evap }}$ in our simple model. We now show that this product is limited by the total scaled atom number $N$ (which in the normalization of Eq.(1) is limited to be at most $\frac{1}{d+1}$ ).

We consider two cooling methodologies which we refer to alternatively as "passive" and "active". They refer respectively to holding the $E_{\text {evap }}$ fixed or suddenly reducing it. Unfortunately, in actual experiments underway ${ }^{13}, E_{\text {evap }}$ is varied continuously, so these two cooling methodologies are probably not directly related to current experiments. This choice of cooling methodologies is advantageous analytically since it does allow evaluation of the effects of evaporation without recourse to any dynamical timescales.

In the passive method, the system is held at a fixed $E_{\text {evap }}$ and allowed to cool by evaporation indefinitely. Dynamically, since the emerging fermi surface at $\mu^{*}$ is always a fraction of $E_{\text {evap }}$, the cooling rate is limited by the overall rate of escape, which (at low temperature) is Boltzmann suppressed by a factor of $e^{-\beta(1-\mu *)}$, and by the trap lifetime. However, we reiterate the "kinetic" model we employ analytically encapsulates limits to sympathetic evaporative cooling of the two component fermion system without including "dynamical" effects (such as collisional or trap timescales).

In the active method on the other hand we abruptly lower $E_{\text {evap }}$ to a value at or below our initial $\mu^{*} E_{\text {evap }}$. Call this new evaporation energy $E_{\text {evap }}^{\prime}$. The distribution function is invariant under such a change in $E_{\text {evap }}$. The $\mu$ and $\beta$ values will trivially jump by factors of the evaporative energy scale ratio. Of course, the overall scaled phase space constants that we suppressed in Eq.(1) do go as positive powers of $E_{\text {evap }}$. Dropping $E_{\text {evap }}$ to $E_{\text {evap }}^{\prime}<E_{\text {evap }}$ has the immediate effect of dropping $N$, for example. This corresponds precisely to the statement that the all the particles with $E>E_{\text {evap }}^{\prime}$ leave immediately.

Since $\mu \beta$ measures our progress towards the ground state and is invariant under a sudden drop in $E_{\text {evap }}$, we see that only subsequent evaporation (and rethermalization) of the remaining fermions can result in increases in the product $\mu \beta$. We now show that evaporation after the drop in $E_{\text {evap }}$ does not lead the system substantially closer to the quantum ground state.

To recapitulate, the relevant question in both cooling methodologies considered here is whether the subsequent evaporative cooling leads to a large $\beta \mu$ product.

The evaporation equations above can be integrated numerically for $\beta(\mu)$ by eliminating $N$ from Eq.(3) and Eq.(4). In doing so, one finds that for values of $\mu \neq \mu^{*}$, the change in the dimensionless temperature ratio $\beta$ is generally relatively small, on the order of $\beta$ itself. To get to a nearly degenerate fermi system starting far away from the ground state, we need a cooling regime in which much larger temperature drops are achievable. In numerical simulations, one finds that the only large temperature changes happen evaporatively when the system is at a $\mu$ very near $\mu^{*}$, basically within 10 percent of that value. Figure 3 is the integral $\beta(\mu)$ of Eq.(3) and Eq.(4) for two initial conditions, $\left(\mu_{i}, \beta_{i}\right)=(0,2.5)$ and $(2.8,4.5)$. Trajectories that start at higher initial temperatures (lower $\beta_{i}$ ) remain substantially lower on Figure 3 throughout the entire evaporative trajectory, but do eventually wind along the limit cycle at $\mu^{*}$. towards large $\beta$. Recall that in the active method we are actually starting generally at smaller $\beta_{i}$ than shown. 


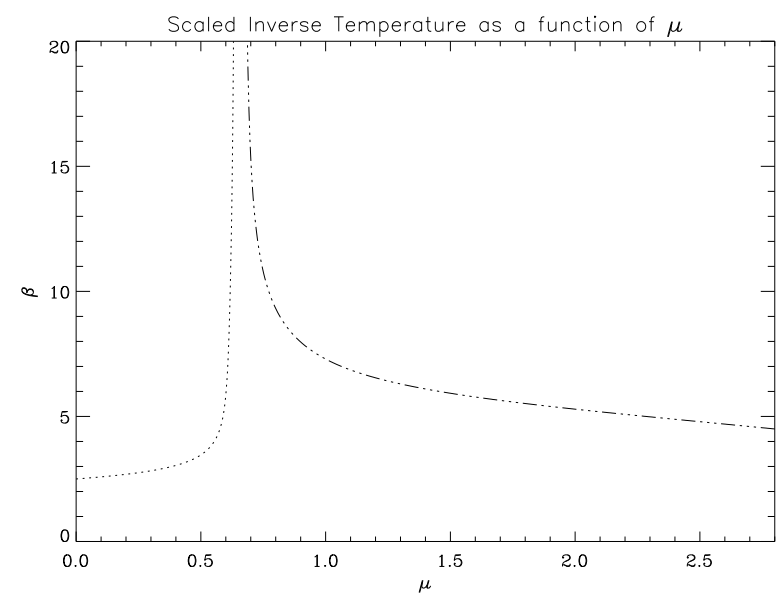

Figure 3: Two Typical Cooling Trajectories, both starting at $\beta \sim 4$, from above and below $\mu^{*}$.

Curiously, note that for $\mu>>\mu^{*}$, the $\mu \beta$ product can actually initially decrease along the evaporative trajectory. In the conclusion we comment on a heuristic way of understanding such counter-intuitive behavior. At any rate, it is clear from Figure 3 that achieving a condensed fermi system requires evolving $\mu$ close to $\mu^{*}$. We now investigate some liberal bounds on how well evaporation can achieve that goal.

In Figure 3 we integrated Eq.(3) and Eq.(4) for $\beta(\mu)$ by eliminating $N$, not taking into account that $N$ must always be decreasing! We now study how far along these $\beta(\mu)$ trajectories we may progress evaporatively until we substantially run out of particles.

Analytically, at low temperature, we find that the flow equation for $\mu$ near $\mu^{*}$ is independent of $\beta$ entirely, and reads,

$$
\frac{\mathrm{d} \mu}{\mathrm{d} N}=\frac{\pi^{2}}{6} \frac{2-3 \mu}{\mu^{3}}+\ldots
$$

which can be integrated for all $\mu$ to read

$$
\left[-\frac{8}{27} \log (\mu-2 / 3)-\mu^{2} \frac{\mu+1}{3}-\frac{4 \mu}{9}\right]_{\mu_{i}}^{\mu_{f}}=\frac{\pi^{2}}{2} \Delta N
$$

The number evaporated $\Delta N$ must of course be less than the total number of particles in the trap. Using Eq.(1), we see that there are indeed stringent limits on the R.H.S. of Eq.(8). Since we know that appreciable cooling in this scheme doesn't occur until one is close to $\mu^{*}(=2 / 3$ at low temperature), we know that the best one can do is to evaporate all the particles in excess of the ground state at $\mu^{*}$. For the scenario in which we start at a $\mu$ above $\mu^{*}$ (for example, as may be created in the active method) this limits the RHS substantially. For $\mu<\mu^{*}$, the system evaporatively evolves towards $\mu^{*}$ but can never reach it because there are simply not enough particles to evaporate. That is one reason to use the active method in a phase of the cooling, since it can raise the initial $\mu$ above the $\mu^{*}$. However, raising $\mu$ by this means is also self-limiting for two reasons. First, if it is raised substantially above 1 too many particles are lost from the trap and there are too few remaining to evaporate back to degeneracy. Secondly, as described earlier, it reduces $\beta_{i}$ by the same factor it increases $\mu_{i}$, indicating the need to get even closer to $\mu^{*}$ to wind along the limit cycle and recover large $\beta$. We explain this in more detail quantitatively below.

We graphically describe the consequences of Eq.(8). All the discussion here is in the "best case" scenario, in that we imagine starting with a system at already relatively large $\beta$ (so Eq.(8) applies) and ask how well evaporative cooling can further increase $\beta$ and thus $\beta \mu$. Figure 4 is a graph of Eq.(8). The dashed line represents a maximum possible RHS at that initial $\mu$. As per the 
preceding discussion we have plotted the contribution from the total number of particles for $\mu<\mu^{*}$, and plot the excess only for $\mu>\mu^{*}$. The light dotted trace is the LHS of Eq.(8). Thus, to estimate the maximum possible increase/decrease in $\mu$, start at some initial $\mu_{i}$, use the height of the dashed line to estimate how much of a change in the height of the light dotted line you may achieve. The resulting position at that height on the light dotted curve then is an (over-)estimate of the largest $\mu$ achievable $\dagger$

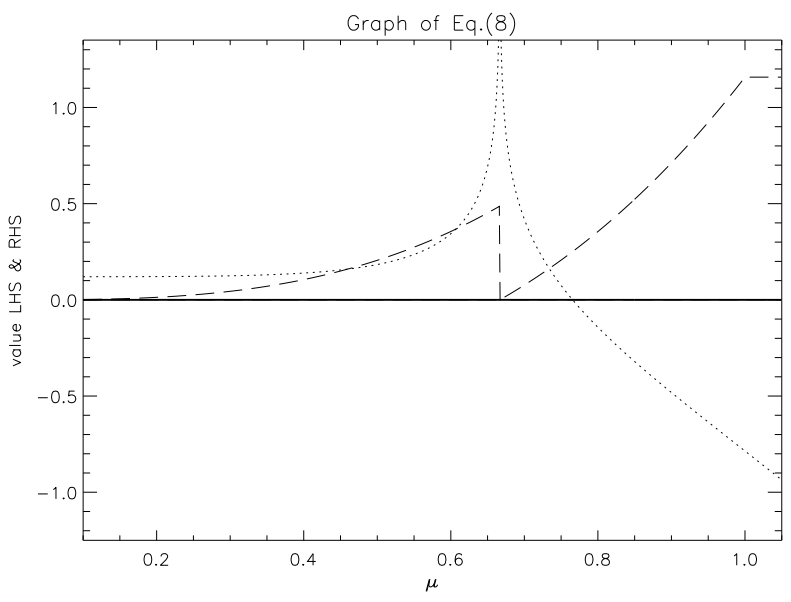

Figure 4: Graph of Eq.(8)

Studying the graph indicates that the process allows one to get within perhaps $10 \%$ of $\mu^{*}$ at best. As a very crude estimate, we can see that this occasions at most a roughly 3 -fold increase in $\beta$. Cooling from a typical active-method initial state of $(\beta, \mu) \sim(1,1)$, we find that one cannot achieve $\beta \mu$ products in excess of roughly 3 . For such a gas, the typical occupation probability of the lowest energy 1-particle state in the trap is roughly $90 \%$. Of course, measuring this occupation probability is not a good measure of quantum degeneracy because it is exponentially sensitive to $\beta \mu$. We prefer thinking about nearly degenerate quantum fermi systems as those with large $\beta \mu$ products such as are found for nucleons in nuclei and electrons in typical metallic systems.

III: A Second Model: We now study the scenario in which the evaporative thresholds for the two species are very different. We forgo a detailed quantitative analysis specific for this case, and instead reduce to and reason from the simpler model we have already discussed. For simplicity we assume that the masses and the trap potential of the two species are identical. Thus, the quasi-equilibrium expectation values of $N_{a, b}$ and $E_{a, b}$ are given by the obvious doubling- and indexing- of Eq.(1). However to include the fact that the evaporative threshold for species $a$ to be so much larger than that of species $b$ replace the upper limit ' 1 ' of the integrals in the Eq.(1) by ' $\infty$ '. We then scale $\beta, \mu_{a}$ and $\mu_{b}$ by the evaporative threshold of the $b$ species. Subsequently $E_{\text {evap }}$ refers to the evaporative threshold for species $b$ only.

The condition, $\mathrm{d} N_{a}=0$ now becomes a linear constraint in the space $\left(\mu_{a}, \mu_{b}, \beta\right)$. All these variables are dimensionless, scaled by the appropriate factors of $E_{\text {evap }}$.

That linear constraint reduces the evaporative evolution of this two-component fermi system again to a two-dimensional dynamical system (i.e. at fixed $E_{\text {evap }}$ and $N_{a}, \mu_{a}$ is really a function of $\beta$ ). In particular, scattering events that leads to evaporation of a particle of species $b$ at energy $E_{\text {evap }}$ will, on average, remove a net $E_{a}$ from the total energy of species $a$ and $E_{b}$ from species $b$,

$\dagger$ Note that although $\left.\frac{\partial N}{\partial \mu}\right|_{\beta}$ is always greater than zero, the total derivative $\frac{\mathrm{d} N}{\mathrm{~d} \mu} \mid<0$ for $\mu<\mu^{*}$ because $\left.\frac{\partial N}{\partial \beta}\right|_{\mu} \frac{\mathrm{d} \beta}{\mathrm{d} \mu}$ is negative. 
where $E_{a}+E_{b}=E_{\text {evap }}$ and with ratio $E_{a} / E_{b}$ depending on $\left(\mu_{a}, \mu_{b}, \beta\right)$. In equations, $\mathrm{d} N_{a}=0$ implies that

$$
\frac{\mathrm{d} E_{a}}{\mathrm{~d} \beta}=-\frac{\operatorname{det}\left(M_{a}\right)}{\left.\frac{\partial N_{a}}{\partial \mu_{a}}\right|_{\beta}}
$$

(compare to Eq.(6)) where $\operatorname{det}\left(M_{a}\right)$ is the determinant of the matrix of partial derivatives for the $a$ system only. Note that this determinant $\operatorname{det}\left(M_{a}\right)$ is now computed with the integrals extending to $\infty$, and so is singular at high temperatures, but still looks like the rest of the graph in Figure 1 for low temperatures. The analogous function for the $b$ species, $\operatorname{det}\left(M_{b}\right)$, is precisely the same as for Eq.(5) with Eq.(1) (that is, using integration limits [0:1]).

Energy conservation implies (in scaled dimensionless quantities) that the evaporative trajectory is along

$$
\left(\begin{array}{c}
\mathrm{d} N_{b} \\
\mathrm{~d}\left(E_{a}+E_{b}\right)
\end{array}\right)=\left(\begin{array}{l}
-1 \\
-1
\end{array}\right) \mathrm{d} N_{b}
$$

The differential relations between $E_{b}, N_{b}, \mu_{b}$ and $\beta$ are exactly the same as for the matrix system analyzed in the first model. We now use Eq.(9) to rewrite the $\mathrm{d} E_{b}$ term in Eq.(10) in terms of $\mathrm{d} \beta$ and use it to re-write the two dimensional system for the evolution of $\mu_{b}$ and $\beta$ in terms of $\mathrm{d} N_{b}$. Recall that $\mu_{a}$ also changes, but is given parametrically in terms of $\beta$ (and $N_{a}$, which is held fixed). We find,

$$
\begin{gathered}
\frac{\partial \beta}{\partial N_{b}}=\frac{1}{\operatorname{det}(\mathcal{M})}\left(-\left.\frac{\partial E_{b}}{\partial \mu_{b}}\right|_{\beta}+\left.\frac{\partial N_{b}}{\partial \mu_{b}}\right|_{\beta}\right) \\
\frac{\partial \mu_{b}}{\partial N_{b}}=\frac{1}{\operatorname{det}(\mathcal{M})}\left(\left.\frac{\partial E_{b}}{\partial \beta}\right|_{\mu_{b}}-\left.\frac{\partial N_{b}}{\partial \beta}\right|_{\mu_{b}}-\frac{\operatorname{det}\left(M_{a}\right)}{\left.\frac{\partial N_{a}}{\partial \mu_{a}}\right|_{\beta}}\right)
\end{gathered}
$$

The denominator is

$$
\operatorname{det}(\mathcal{M})=\operatorname{det}\left(M_{b}\right)+\operatorname{det}\left(M_{a}\right)\left(\frac{\left.\frac{\partial N_{b}}{\partial \mu_{b}}\right|_{\beta}}{\left.\frac{\partial N_{a}}{\partial \mu_{a}}\right|_{\beta}}\right)
$$

and is again strictly positive.

We can now use the intuition based on the first model discussed in detail above to constrain how well one can cool the $a$ species in this scenario. The measure of how close to the ground state we are for that species is again the product $\beta \mu_{a}$. Consider two possible initial high temperature limits: $N_{a}>>N_{b}$ and $N_{a}<<N_{b}$. In the first case, $\mu_{a}$ is high but there are fewer $b$ species to evaporate off, and by the analysis of the first model, we expect only a modest increase in $\beta$. In the other extreme, $\mu_{a}$ starts off small, and so, although in principle you could carry off much heat, it's temperature will track the temperature of the $b$ species, which will again be limited by all the considerations discussed in the first model, and so the product $\beta \mu_{a}$ will start small and stay small. Thus, the most promising initial (high temperature) state is $N_{a} \sim N_{b}$ (that is, $\mu_{a} \sim \mu_{b}$ ).

Looking now at Eq.(12), we see that the limit cycle still exists and occurs at a value $\mu_{b}^{*}$ lower than in the first model we discussed. Also note that the $\beta$ evolution equation in this scenario differs only by the prefactor, $\operatorname{det}(\mathcal{M})$, from the first model we considered. Due to positivity of the individual $\operatorname{det}\left(M_{a, b}\right)$, we expect that factor to reduce the evolution of $\beta$ as compared with the first model. Indeed, in the high temperature limit the suppression is through powers of the ratio of the evaporative energy scales of the two species, but for low temperatures, $\operatorname{det}(\mathcal{M}) \sim 2 \operatorname{det}\left(M_{b}\right)$. Thus, as may have been expected due to the larger thermal inertia of the entire system compared with that of an individual species, the actually overall evaporative cooling efficiency is suppressed at low temperatures relative to the first model we considered. 
This means that if we cool in the active mode, $\mu_{b}$ will be large initially and thus the temperature of the entire system will not drop dramatically as one evaporates. Efficient cooling could occur if we are able to get to $\mu \sim \mu^{*}$, but it is difficult to reach that regime because, although we can evaporate all of species "b" (thereby having a continuous curve for the dashed curve in Figure 4 representing the upper bound for the RHS of Eq.(8)), in the low temperature limit the cooling of the whole system proceeds slower, and so the light dotted curve in Figure 4 is roughly twice as steep. Thus, the obstruction to reaching the quantum ground state in the two component system in this regime (unequal $E_{\text {evap }}$ ) can be understood from the considerations and qualitative behavior of the first regime (equal $E_{\text {evap }}$ ).

IV: Conclusion: There exists a limit cycle in evaporative cooling a two component fermion system that has the consequence of severely limiting the approach to the quantum degenerate ground state. One heuristic way to understand this result is that since there is always a gap between the putative fermi surface and the evaporative threshold, evaporation can actually "heat up" (that is, disorder) the distribution function at the fermi surface. This one way to understand the rather counterintuitive finding that that starting at $\mu>>\mu^{*}$, the $\mu \beta$ product actually initially decreases during evaporation.

There are many obvious reasons why the two component fermi system is so different than the bose system. From the point of view of the discussion here, the trapped bose gas starts with $\mu<<0$ and, analogous to what we have been discussing, evaporatively evolves towards a limit cycle at some $\mu^{*}>0$. But, of course due to the singularity of the system at $\mu \rightarrow 0^{-}$, it never gets to $\mu^{*}$ but instead condenses.

There have been many proposals for surmounting the difficulty of achieving a degenerate fermi ground state, and it is not the purpose of this note to review these many inventive ideas. They include condensing fermi-bose mixtures (a difficult technical feat) $14,15,16,17,19$ and using various perturbing fields on pure fermi systems. What we suggest is that many cooling techniques that evaporate fermions exclusively may be constrained in the same way as described above.

We would like to end with a brief speculative proposal for reaching lower $\beta \mu$ products in a trapped atomic fermi gas. Consider photoassociating fermi dimers into states just below the trap single particle ground state. With fermions there is no stimulated atomic channel back to the trap as would the case of photoassociating dimers from a bose condensate; instead pauli blocking and the fermi energy both push the system towards dimerization. The remaining fermions then scatter off the dimers. In a sense, photoassociating has enhanced the three-body collision rate, which, even for identical fermions, is not suppressed by statistics at low energies. Every time a dimer breaks in collision, as long as the trapping potential is high enough, the fermions go back into the trap; the net effect of creating and breaking dimers in this proposed scheme is to use the difference in the dimer pump beams to cool the fermion system "from below" (near the single particle ground state of the trap) instead of evaporatively "from above" (that is, above the fermi surface). In that sense this scheme has the flavor of bose-fermi mixture schemes, but might be simpler technically. Also, this cooling proposal does not a priori require a two fermion mixture, though we imagine photoassociating into a dimer composed of dissimilar fermions is likely to be easier than in identical ones. It remains to be seen whether such a technique can be practically implemented in a polarized atomic fermi system.

Finally, it would be of great interest to compare the predictions of this simple evaporative model directly with experiment. One step in that direction is to generalize the model to include a time dependent $E_{\text {evap }}$ and trap lifetime effects. One use of such a direct comparison would be to further test how large quantum statistical effects are in current experiments which are far from degeneracy. Investigations of such "dynamical" effects are underway but clearly beyond the "kinematic" scope 
and spirit of this note.

V: Acknowledgments It is a pleasure to thank D. S. Jin, R. Walsworth and W. Phillips for stimulating conversations and suggestions. We also thank J. Baltisberger for numerical work leading to Figure 1. This research was supported in part by Research Corporation Cottrell Science Award \#CC3943 and in part by the National Science Foundation under grants PHY 94-07194 and EPS9874764 . 


\section{Bibliography}

1. M. H. Anderson, J. R. Ensher, M. R. Mathews, C. E. Weiman and E. A. Cornell, Science 269, 198 (1995).

2. K. B. Davis et. al., Phys. Rev. Lett. 75, 3969 (1995).

3. C. C. Bradley, C. A. Sackett and R. G. Hulet, (to be published).

4. I. Silvera, Physica 109 \& 110B (1982) 1499-1522.

5. J. Oliva, Phys. Rev. B 38, 8811 (1988) and Phys. Rev. B 39, 4204 (1989).

D. A. Butts and D. Rokhsar, Phys. Rev A 554346 (1997).

6. H. T. C. Stoof and M. Houbiers, cond-mat/9808171.

7. B. DeMarco and D. S. Jin, Phys. Rev. A 58, R4267 (1998).

8. G. Brunn, Y. Castin, R. Dum, K. Burnett, cond-mat/9810013.

9. B. DeMarco, Bohm, Burke, Holland, D. S. Jin, Phys. Rev. Lett., 82:(21) 4208, (1999), condmat/9812350.

10. C. J. Myatt, E. A. Burt, R. W. Ghrist, E. A. Cornell and C. E. Weiman, Phys. Rev. Lett. 78, 586 (1997).

11. G. Ferrari, Phys. Rev. A, 59:(6) R4125, (1999), cond-mat/9904162.

12. W. Geist, A. Indrizbecovic, M. Marinescu, T. A. B. Kennedy and L. Youm, cond-mat/9907222.

13. D. S. Jin, Private Communication.

14. N. Nygaard and K. Moelmer, cond-mat/9901160.

15. L. Vichi, M. Ingusclo, S. Stringari and G. M. Tino, J. Phys. B - AT MOL OPT 31:(21) L899,(1998), cond-mat/9810115.

16. M. Amoruso, A. Minguzzi, S. Stringari, M. P. Tosi and L. Vichi, Eur. Phys. J. D. 4:(3) 261, (1998), cond-mat/9810210.

17. T. Miyakawa, K. Oda, T. Suzuki and H. Yabu, cond-mat/9907009

18. L. Vichi and S. Stringari, cond-mat/9905154.

19. W. Geist, L. You and T. A. B. Kennedy, Phys. Rev. A 59:(2) 1500, (1999). 